\title{
Isolation and Identification of Rhizospheric Bacteria in Sugarcane (Saccharum spp. L.) Cultivated on Acrisols of Tay Ninh Province, Vietnam
}

\author{
Hoang Minh Tam \\ Dept. Natural Science Teacher Training, Sai Gon University, HCM City, Vietnam \\ Cao Ngoc Diep \\ Dept. Microbiology Biotechnology, Biotechnology R\&D Institute, Can Tho University, Can Tho City, Vietnam
}

\begin{abstract}
Nitrogen-fixing and phosphate-solubilizing bacterial diversity and population dynamics in the Acrisol rhizosphere of sugarcane grown in Tay Ninh province, the South-Eastern Vietnam was studied. Soil rhizosphere samples were taken in seven districts (sites) of this region. Physical and chemical characteristics of the soil samples as well as total nitrogen-fixing and phosphate-solubilizing bacteria counts were determined by drop plate method together with $16 \mathrm{~S}$ rRNA gene fragments amplified from DNA using eubacterial universal primers (27F and 1492R). A total of 331 isolates were isolated on two media (Burk's N-free and NBRIP) and all of them have ability of nitrogen fixation and phosphate solubilization together with IAA biosynthesis. Population of nitrogen-fixing and phosphate-solubilizing bacteria correlated with organic matter content in soil very closely $(\mathrm{P}<0.01)$ and phosphate-solubilizing baceria population in soil related with available $\mathrm{P}_{2} \mathrm{O}_{5}$ very closely $(\mathrm{P}<0.01)$ in soil. The sequences from selected nitrogen-fixing and phosphatesolubilizing bacteria (23 isolates) showed high degrees of similarity to those of the GenBank references strains (between 97\% and 100\%). From 23 isolates, 6 strains belonged to Bacilli, while 4 strains were Beta-Proteobacteria, and 13 strains were Gamma-proteobacteria. Based on Pi value (nucleotide diversity), Gammaproteobacteria group had the highest theta value and Theta values (persequence) from S of SNP for DNA polymorphism were calculated for each group and Gammaproteobacteria group had the highest values in comparison with two groups. From these results showed that four strains (Burkholderia sp. DMC5e, Bacillus subtilis GOD1c, Bacillus subtilis CHT1d and Burkholderia sp. TAC3b) revealed promising candidates with multiple beneficial characteristics and they have the potential for application as inoculants adapted to poor soils and sugarcane because they are not only famous strains but also safe strains for sustainable agriculture.
\end{abstract}

Keywords - Acrisols, 16S rRNA Gene Sequence, Nitrogen-Fixing Bacteria, Phosphate-Solubilizing Bacteria, Rhizosphere, Sugarcane

\section{INTRODUCTION}

Sugarcane (Saccharum sp. L.) is grown in more than 120 countries, mainly in Brazil and India [1] and Vietnam with 283,000 ha in which the South-Eastern Vietnam occupied 34,395 ha and Tay Ninh province had 29,287 ha (86\% among 34,395 ha) in 2014 [2]. It has been a general practice to apply $250 \mathrm{~kg} \mathrm{~N}$ ha-1 yr-1 or more than in most sugarcane cultivating countries [3] and Vietnamese farmers want to have a high sugarcane yield (over 200 tons/ha), they should apply high quantity of inorganic nitrogen fertilizer (urea) (approx. $200 \mathrm{~kg} \mathrm{~N} \mathrm{ha-1} \mathrm{yr-1,} 100 \mathrm{~kg} \mathrm{P}_{2} \mathrm{O}_{5}$ ha-1 (equivalent $400 \mathrm{~kg}$ superphosphate $15 \% \mathrm{P}_{2} \mathrm{O}_{5}$ ha-1) and $150 \mathrm{~kg} \mathrm{~K} \mathrm{~K}_{2} \mathrm{O}$ ha-1 (equivalent $250 \mathrm{~kg} \mathrm{KCl} 60 \% \mathrm{~K} \mathrm{O}_{2} \mathrm{Oa}-1 \mathrm{yr}-$ 1)[4].].

The Tay Ninh province, Vietnam locates from $105^{\circ} 48^{\prime} 43^{\prime \prime}$ to $106^{\circ} 22^{\prime} 48^{\text {ee }} \mathrm{E}$ and from $10^{\circ} 57^{\prime} 08^{\mathrm{ee}}$ to $11^{\circ} 06^{\prime} 16^{\mathrm{ee}}$ $\mathrm{N}$, it is located one of the two regions of South Vietnam situated in the East of South Vietnam. The soils are mainly acrisols with a $\mathrm{pH}$ range of $3.98-4.56$. They are considered nutrient poor, with an average organic matter of $<1 \%$, a total nitrogen range of $0.07-0.11 \%$, and a very low available phosphorus, cation exchange capacity, exchangeable $\mathrm{K}$ and contain more sand in their structure [5] (Table 1). 
Table -1 Acrisols in Tay Ninh province [5]

\begin{tabular}{llcc}
\hline & \multicolumn{1}{c}{ Treatment } & Acrisols on alluvial soil & Gley acrisols on alluvial soil \\
\hline 1 & Structure (\%): & sandy & sandy \\
2 & Total CEC (meq/100g): low & $54.87-61.43$ & $51.53-61.06$ \\
3 & $\mathrm{pH}_{\mathrm{KCl}}$ : low & $2.14-2.32$ & $1.48-2,03$ \\
4 & Organic matter C (\%): low & $3.98-4.22$ & $4.27-4.56$ \\
5 & $\mathrm{~N}$ total (\%): low & $0.91-1.01$ & $0.49-0.72$ \\
6 & Available $\mathrm{P}_{2} \mathrm{O}_{5}(\mathrm{mg} / 100 \mathrm{~g}$ soil): & $0.07-0.09$ & $0.08-0.11$ \\
7 & Kali $\mathrm{K}_{2} \mathrm{O}$ total (\%): low & $1.07-1.12$ & $1.27-1.55$ \\
8 & Kali $\mathrm{K}_{2} \mathrm{O}$ exchangeable (mg/100g soil): low & $0.17-0.19$ & $0.24-0.27$ \\
\hline
\end{tabular}

The narrow zone of soil directly surrounding the root systems is referred to as rhizosphere [6], while the term "rhizobacteria" implies a group of rhizosphere bacteria component in colonizing the root environment [7]. Plant growth promoting rhizobacteria are the soil bacteria inhabiting around / or on the root surface and are directly or indirectly involved in promoting plant growth and development via production and secretion of various regulatory chemicals in the vicinity of rhizosphere [8].

In order to make sustainable sugarcane cultivation and less dependent on chemical nitrogen fertilizers, it needs to be found the proportion of plant promoting bacteria, which are PGPR. The aims of this study were (i) isolating of rhizospheric bacteria, (ii) studying characteristic such as nitrogen fixation, phosphate solubilization and IAA production, (iii) evaluating the genetic diversity of PGPR isolated from soil in order to identify efficient growth promotion strains that can also improve the growth of sugarcane plant as biofertilizer.

\section{MATERIALS AND METHODS}

\section{A. Soil Sample and Isolation of Bacteria}

The sugarcane plants were sampled at the stage of plant having 6 month-old (June-July 2016) from the fields of the districts of Tay Ninh province)[map determined the locates where collected samples were presented in Figure 1.

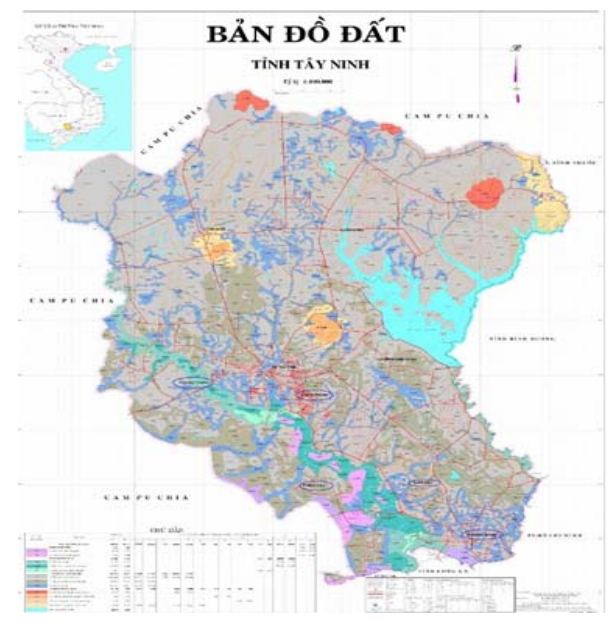

Figure 1: The locations were examined in this study and sugarcane rhizopheric soil samples were collected at the districts in Tay Ninh province

Rhizospheric soils around sugarcane plants were collected by moving the soil that adhered to the roots (stem and root of sugarcane plant will be used in further experiment) and they were kept in refrigerator for counting viable cells by drop plate method [9] and isolating of nitrogen-fixing bacteria in Burk's $\mathrm{N}$ free medium [10] and phosphatesolubilizing bacteria in NBRIP medium [11]; cultures were streaked on media to obtain single colonies. To check for phosphate solubilizing ability or nitrogen fixating ability, colonies from Burk's $\mathrm{N}$ free medium were streaked to NBRIP medium and colonies from NBRIP medium were also cultivated to Burk's N free medium in order to select 
the colonies which developed on two media (or microbes having both $\mathrm{N}_{2}$-fixing and phosphate-solubilizing abilities).

\section{B. Screening for Biofertilizer Activities}

The ability to fix $\mathrm{N}_{2}$ was tested on Burk's $\mathrm{N}$-free liquid medium incubating at $30^{\circ} \mathrm{C}$ and the ammonium concentration in medium was measured by Phenol Nitroprusside method after 2, 4, 6 and 8 days inoculation (DAI). Besides, inorganic phosphate solubilizing ability was tested on NBRIP liquid medium incubated at $30^{\circ} \mathrm{C}$ and the $\mathrm{P}_{2} \mathrm{O}_{5}$ concentration was measured by ammonium molypdate method. The qualitative detection of indole-3-acetic acid (IAA) production was carried out basing on the colorimetric method [12]. Precultures were grown in Burk's N free $(100 \mathrm{~mL})$ without tryptophan in $250 \mathrm{~mL}$-flask at $30^{\circ} \mathrm{C}$ on a roller at $100 \mathrm{rpm}$ and samples were taken at $2,4,6$, and $8 \mathrm{DAI}$, cell free supernatants were mixed 2:1 with Salkowski reagent $\left(0.01 \mathrm{M} \mathrm{FeCl}_{3}\right.$ in $35 \%$ perchloric acid) and incubated in the dark for $20 \mathrm{~min}$ at RT. IAA-containing solutions were indicated by reddish color with an absorption peak at $530 \mathrm{~nm}$ on Thermo Scientific GENESYS 10Uv spectrophotometer. Furthermore, siderophore production was assayed by the rhizopheric bacterial isolates according to Schwyn and Neilands [13] using NBRIP medium without tryptophan which was diluted fivefold. The isolates were spot inoculated onto Chrome azurol S agar plates divided into equal sectors, and the plates were iincubated at $28^{\circ} \mathrm{C}$ for $48 \mathrm{~h}$. Development of a yellow, orange or violet halo around the bacterial colony was considered to be positive for siderophore production.

Besides that, the $\mathrm{pH}$ of rhizosphere soil was measured in a 1:5 soil to water $(\mathrm{w} / \mathrm{v})$ mixture in $20 \mathrm{~min}$ and read on a Jenway $3510 \mathrm{pH}$ meter, $\mathrm{N}$ total was measured using the micro-Kjeldahl digestion method, the colorimetric $\mathrm{P}$ determination was based on the method of ammonium molypdate method [14] and organic carbon measured by Walkley-Black method [15].

\section{C. $16 \mathrm{~S}$ rDNA Gene Amplification and Sequencing}

Bacterial DNA was isolated following published protocols [16]; Amplification of 16S rDNA by PCR was carried out using the universal primers $27 \mathrm{~F}$ and $1492 \mathrm{R}$ [17]. The $50 \mu \mathrm{L}$ reactionmixture consisted of $2.5 \mathrm{U}$ Taq Polymerase (Fermentas), $50 \mu \mathrm{M}$ of each deoxynucleotide triphosphates, $500 \mathrm{nM}$ of each primer (Fermentas) and $20 \mathrm{ng}$ DNA. The thermocycling profile was carried out with an initial denaturation at $95^{\circ} \mathrm{C}(5 \mathrm{~min})$ followed by 30 cycles of denaturation at $95^{\circ} \mathrm{C}(30 \mathrm{~s})$, annealing at $55^{\circ} \mathrm{C}(30 \mathrm{~s})$, extension at $72^{\circ} \mathrm{C}(90 \mathrm{~s})$ and a final extension at $72^{\circ} \mathrm{C}(10 \mathrm{~min})$ in C1000 Thermal Cycler (Bio-Rad).

Aliquots $(10 \mu \mathrm{l})$ of PCR products were separated and visualized in $1 \%$ agarose gels by using standard electrophoresis procedures. Partial 16S rRNA genes of selected isolates in each group were sequenced by MACROGEN, Republic of Korea (dna.macrogen.com). Finally, 16S rRNA sequence of the isolate was compared with that of other microorganisms by way BLAST_http://www.ncbi.nlm.nih.gov/BLAST/Blast.cgi). In the best isolate(s) (high nitrogen fixing and phosphate solubilizing ability) and 12 isolates of 3 sites were chosen to sequence and the results were compared to sequences of GenBank based on partial 16S rRNA sequences to show relationships between PGPR strains [18] and phylogenetic tree was constructed by the maximum-likelihood method using the MEGA software version 6.06 based on 1000 bootstraps.

\section{SNPs Discovery}

The sequence date from 24 root-associated bacterial isolates were analysed with SeqScape@Software (Applied Biosystem, Foster City, CA, USA). SeqScape is a sequence comparison tool for variant identification, SNP discovery and validation. It considers alignment depth, the base calls in each of the sequences and the associated base quality values. Putative SNPs were accepted as true sequence variants if the quality value exceeded 20. It means a $1 \%$ chance basecall is incorrect.

\section{E. Nucleotide Diversity $(\theta)$}

Nucleotide diversity $(\Theta)$ was calculated by the method described by Halushka et al. [19]

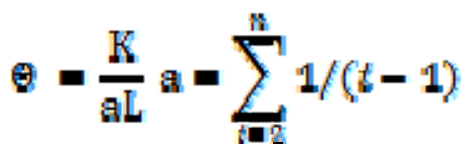

where $\mathrm{K}$ is the number of SNPs identified in an alignment length, $\mathrm{n}$ is alleles and $\mathrm{L}$ is the total length of sequence (bp). 


\section{F. Data Analyses}

Relationship between population of nitrogen-fixing and phosphate-solublilizing bacteria and soil $\mathrm{pH}, \mathrm{N}$ total, available $\mathrm{P}$ and organic matter content in acrisols were explored with simple regressions using Exel in Microsoft version 7.0. Data from ammonium, orthophosphate and IAA concentrations in media were analysed in completely randomized design with three replicates and Duncan test at $\mathrm{P}=0.01$ and $\mathrm{P}=0.05$ were used to differentiate between statistically different means using SPSS version 16.

\section{RESULTS AND DISCUSSION}

\section{A. Soil Characteristics}

Seven districts (sites) in Tay Ninh province (in eastern of South Vietnam) have cultivated large sugarcane area (Tan Chau, Duong Minh Chau and Tan Bien) and sugarcane almost has been cultivated on Acrisols, this showed that characteristic of acrisol is low soil $\mathrm{pH}$, organic matter content together with low available $\mathrm{P}_{2} \mathrm{O}_{5}$ content $($ Table 1). Interestingly, nitrogen - fixing bacterial population and phosphate-solubilizing bacterial population in acrisols were high (almost over one million cells per dry soil gram) but population of phosphate-solubilizing bacteria in acrisols was low (Table 2) and soil $\mathrm{pH}, \mathrm{N}$ total, available phosphorus also were high in comparison to analysis of Duong [5] perhaps farmers applied lime and/or phosphate fertilizer in crop cultivation and this results led to high nitrogen-fixing and phosphate-solubilizing bacterial population in acrisols.

Table - 2 Soil characteristics and $\mathrm{N}_{2}$-fixing and Phosphate-solubilizing bacterial population in acrisols rhizosphere

\begin{tabular}{|c|c|c|c|c|c|c|c|}
\hline No & Soil sample site & $\mathrm{pH}$ & $\begin{array}{l}\mathrm{N} \\
\text { total } \\
(\%) \\
\end{array}$ & $\begin{array}{l}\text { Avalable } \mathrm{P}_{2} \mathrm{O}_{5} \\
(\mathrm{mg} / 100 \mathrm{~g} \text { soil })\end{array}$ & $\begin{array}{l}\text { Organic matter } \\
\qquad(\%)\end{array}$ & $\begin{array}{c}\mathrm{N}_{2} \text {-fixing bacteria } \\
\text { population } \\
\text { CFU/g soil }\end{array}$ & $\begin{array}{c}\text { Phosphate-solubilizing } \\
\text { bacteria population } \\
\text { CFU/g soil }\end{array}$ \\
\hline \multirow[t]{5}{*}{01} & Trang Bang district & & & & & & \\
\hline & Gia Binh & 6.71 & 0.209 & 1.89 & 3.33 & 6.255 & 6.903 \\
\hline & Loc Hung & 6.77 & 0.070 & 4.41 & 2.77 & 6.505 & 7.903 \\
\hline & Gia Loc & 6.61 & 0.138 & 4.73 & 2.97 & 7.881 & 7.093 \\
\hline & Hung Thuan & 6.75 & 0.139 & 5.61 & 5.11 & 6.643 & 8.193 \\
\hline \multirow[t]{9}{*}{02} & Chau Thanh district & & & & & & \\
\hline & An Binh & 6.81 & 0.069 & 4.27 & 1.97 & 6.079 & 5.778 \\
\hline & Hao Duoc a & 6.71 & 0.205 & 3.11 & 4.22 & 6.991 & 8.049 \\
\hline & An Binh & 6.50 & 0.139 & 1.81 & 1.85 & 6.978 & 6.919 \\
\hline & Hao Duoc b & 6.65 & 0.279 & 3.92 & 1.71 & 6.000 & 5.301 \\
\hline & An Co a & 6.81 & 0.070 & 2.61 & 4.86 & 7.459 & 9.723 \\
\hline & $\mathrm{An} \mathrm{Co} \mathrm{b}$ & 6.85 & 0.138 & 3.48 & 2.02 & 6.681 & 6.415 \\
\hline & Dong Khoi & 6.55 & 0.139 & 3.34 & 4.61 & 9.369 & 8.465 \\
\hline & Cay Xieng & 6.36 & 0.203 & 1.56 & 3.92 & 8.342 & 8.447 \\
\hline \multirow[t]{7}{*}{03} & Go Dau district & & & & & & \\
\hline & Thanh Phuoc & 6.67 & 0.070 & 1.33 & 2.51 & 6.681 & 6.079 \\
\hline & Phuoc Trach a & 6.67 & 0.208 & 1.58 & 2.57 & 6.663 & 6.342 \\
\hline & Phuoc Trach b & 6.81 & 0.138 & 1.42 & 2.36 & 6.079 & 6.255 \\
\hline & Hiep Thanh & 6.70 & 0.152 & 1.14 & 2.45 & 6.653 & 6.491 \\
\hline & Thanh Phuoc & 6.76 & 0.070 & 2.26 & 3.48 & 6.663 & 6.447 \\
\hline & Phuoc Dong & 6.55 & 0.068 & 2.05 & 2.63 & 6.914 & 6.792 \\
\hline \multirow[t]{7}{*}{04} & Ben Cau district & & & & & & \\
\hline & An Thanh & 6.24 & 0.102 & 3.45 & 1.89 & 6.687 & 6.147 \\
\hline & Loi Thuan & 6.66 & 0.128 & 3.02 & 2.14 & 6.647 & 6.664 \\
\hline & Long Giang & 6.47 & 0.139 & 3.55 & 2.55 & 6.125 & 6.258 \\
\hline & Long Phuoc a & 6.58 & 0.147 & 3.39 & 2.98 & 6.258 & 6.587 \\
\hline & Long Phuoc b & 6.84 & 0.155 & 2.96 & 2.87 & 6.589 & 6.894 \\
\hline & Long Chu & 6.33 & 0.168 & 3.49 & 2.54 & 6.478 & 6.877 \\
\hline \multirow[t]{9}{*}{05} & $\begin{array}{lll}\begin{array}{l}\text { Dương } \\
\text { district }\end{array} & & \\
\end{array}$ & & & & & & \\
\hline & Truong Mit a & 6.77 & 0.112 & 5.44 & 1.91 & 7.177 & 6.352 \\
\hline & Truong Mit b & 6.44 & 0.148 & 4.80 & 2.34 & 6.505 & 7.001 \\
\hline & Phuoc Ninh & 6.49 & 0.119 & 2.12 & 2.65 & 6.352 & 6.381 \\
\hline & Cha La a & 6.35 & 0.157 & 2.01 & 2.98 & 6.741 & 6.113 \\
\hline & Cha La b & 6.32 & 0.125 & 1.25 & 2.89 & 5.741 & 6.061 \\
\hline & Phan a & 6.77 & 0.178 & 1.04 & 2.94 & 6.607 & 5.392 \\
\hline & Phan b & 6.36 & 0.123 & 2.77 & 2.64 & 6.691 & 7.251 \\
\hline & Phuoc Minh a & 6.64 & 0.213 & 2.01 & 2.14 & 6.227 & 6.531 \\
\hline
\end{tabular}




\begin{tabular}{|l|l|c|c|c|c|c|c|}
\hline & Phuoc Minh b & 6.34 & 0.133 & 1.12 & 1.22 & 6.531 & 5.778 \\
\hline 06 & Tan Chau district & & & & & & \\
\hline & Thanh Đong & 6.59 & 0.108 & 1.43 & 1.22 & 6.525 & 6.021 \\
\hline & Tan Hung & 6.93 & 0.094 & 1.27 & 1.31 & 6.462 & 6.097 \\
\hline & Tan Phu & 6.98 & 0.099 & 1.28 & 1.28 & 6.466 & 6.112 \\
\hline 07 & Tan Bien district & & & & & & \\
\hline & Tra Vong & 6.49 & 0.123 & 1.54 & 1.47 & 6.321 & 6.588 \\
\hline & Mo Cong & 6.51 & 0.124 & 1.53 & 1.47 & 6.322 & 6.585 \\
\hline & Tan Phong & 7.08 & 0.211 & 1.46 & 1.08 & 6.607 & 5.176 \\
\hline & Thach Binh & 7.05 & 0.212 & 1.47 & 1.09 & 6.611 & 5.188 \\
\hline & Thanh Binh & 7.11 & 0.215 & 1.51 & 1.11 & 6.621 & 5.212 \\
\hline
\end{tabular}

The results from Table 3 showed that there was no significant linear relationship between population of $\mathrm{N}_{2}$-fixing and phosphate-solubilizing bacteria and soil $\mathrm{pH}$ while both microbes organic matter content showed a linear relationship significant at $\mathrm{P}<0.01\left(\mathrm{y}=0.3423 \mathrm{x}+5.8017, \mathrm{r}=0.449^{* *} ; \mathrm{y}=0.7247 \mathrm{x}+4.8158, \mathrm{r}=0.765^{*}\right.$, respectively).

Table - 3 The relationship between population of $\mathrm{N}_{2}$-fixing and phosphate-solubilizing bacteria with $\mathrm{pH}, \mathrm{N}$ total, available phosphorus and organic matter content in soil

\begin{tabular}{|l|l|l|}
\hline \multirow{2}{*}{ Characteristic } & \multicolumn{1}{|c|}{ Population (cfu/dry soil gramme) } \\
\cline { 2 - 3 } Soil $\mathrm{pH}$ & \multicolumn{1}{|c|}{ Nitrogen-fixing bacteria } & \multicolumn{1}{|c|}{ Phosphate-solubilizing bacteria } \\
& $\mathrm{r}=0.075^{\mathrm{ns}}$ & $\mathrm{r}=0.135^{\mathrm{ns}}$ \\
$\mathrm{N}$ total $(\%)$ & $\mathrm{Y}=-0.2189 \mathrm{X}+8.1414$ & $\mathrm{Y}=0.5917 \mathrm{X}+10.506$ \\
& $\mathrm{Y}=-0.3726 \mathrm{X}+6.4619$ & $\mathrm{r}=0.30 *$ \\
& $\mathrm{Y}=0.176^{\mathrm{ns}}$ & $\mathrm{r}=0.423 * *$ \\
Available $\mathrm{P}\left(\mathrm{mg} \mathrm{P}_{2} \mathrm{O}_{5} / 100 \mathrm{~g}\right.$ soil) & $\mathrm{Y}=0.0889 \mathrm{X}+6.4619$ & $\mathrm{Y}=0.3026 \mathrm{X}+5.8866$ \\
\hline Organic matter $(\%)$ & $\mathrm{r}=0.449^{* *}$ & $\mathrm{r}=0.765 * *$ \\
& $\mathrm{Y}=0.3423 \mathrm{X}+5.8017$ & $\mathrm{Y}=0.7247 \mathrm{X}+4.8158$ \\
\hline
\end{tabular}

Almost their colonies have round-shaped; milky (on Burk's medium) and yellow (on NBRIP medium); entire or lobate margin (Figure 3); diameter size of these colonies varied from 0.2 to $2.5 \mathrm{~mm}$ and all of them are Grampositive and Gram-negative by Gram stain. Especially phosphate-solubilizing bacteria make haloes around colonies in NBRIP medium as described of Thanh and Diep [20]. The cells were observed by SEM and appeared as short rods and most of them have motility (Figure 4).

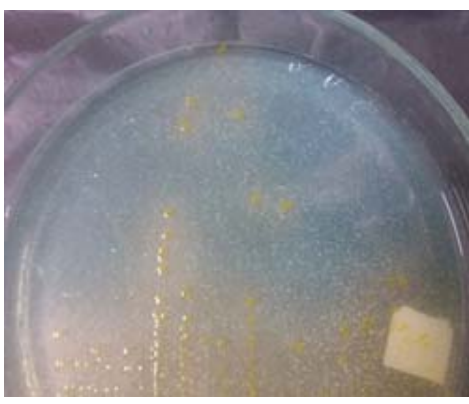

A

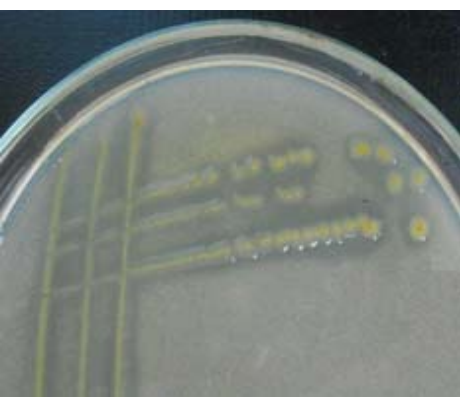

B

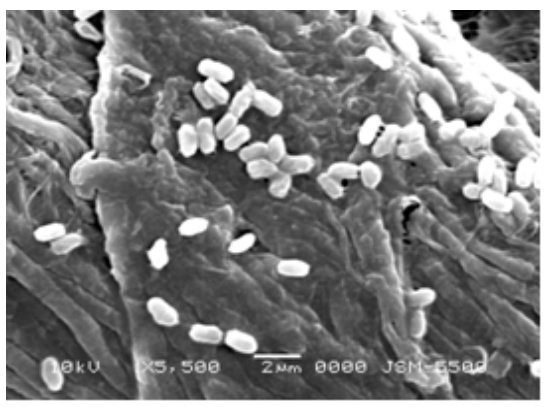

Figure - 3 The colonies of several isolates on NBRIP medium (B) with the halos around the colonies and on Burk's $\mathrm{N}$ free (B)

Figure - 4 Electron micrograph of cells

Among 331 isolates, 112 isolates having good biofertilizer activity were chosen to study (Table 4). Fourty-two isolates had excellent ability of nitrogen fixation in each district (in group of Burk's no N medium) and each district had good isolates as BCA12, CHT1a, CHT1d, DMC5e, GOD2c, TAB5a, TAC3b, TRB4b but the DMC5d isolate had the highest nitrogen fixing ability. In addition, seventy isolates having high abitily of phosphate solubilization were also chosen from seven sites (districts) in Tay Ninh provinve and each site had good isolates as BCA34, BCA37, BCA39, CHT3b, DMC1a, GOD1c, TAC3a, TAC3b, TRB4d, TRB1d (Table 5). In medium without 
tryptophan, all of the isolates produced IAA with low concentration in-vitro. Especially, there were only 24/331 isolates (7.25\%) produced siderophores after 2 days incubated on CAS medium (Figure 5).

Table - 4 Nitrogen fixation $\left(\mathrm{mg} \mathrm{NH}_{4} / \mathrm{ml}\right)$ of 42 isolates (6 isolates/site [or district])

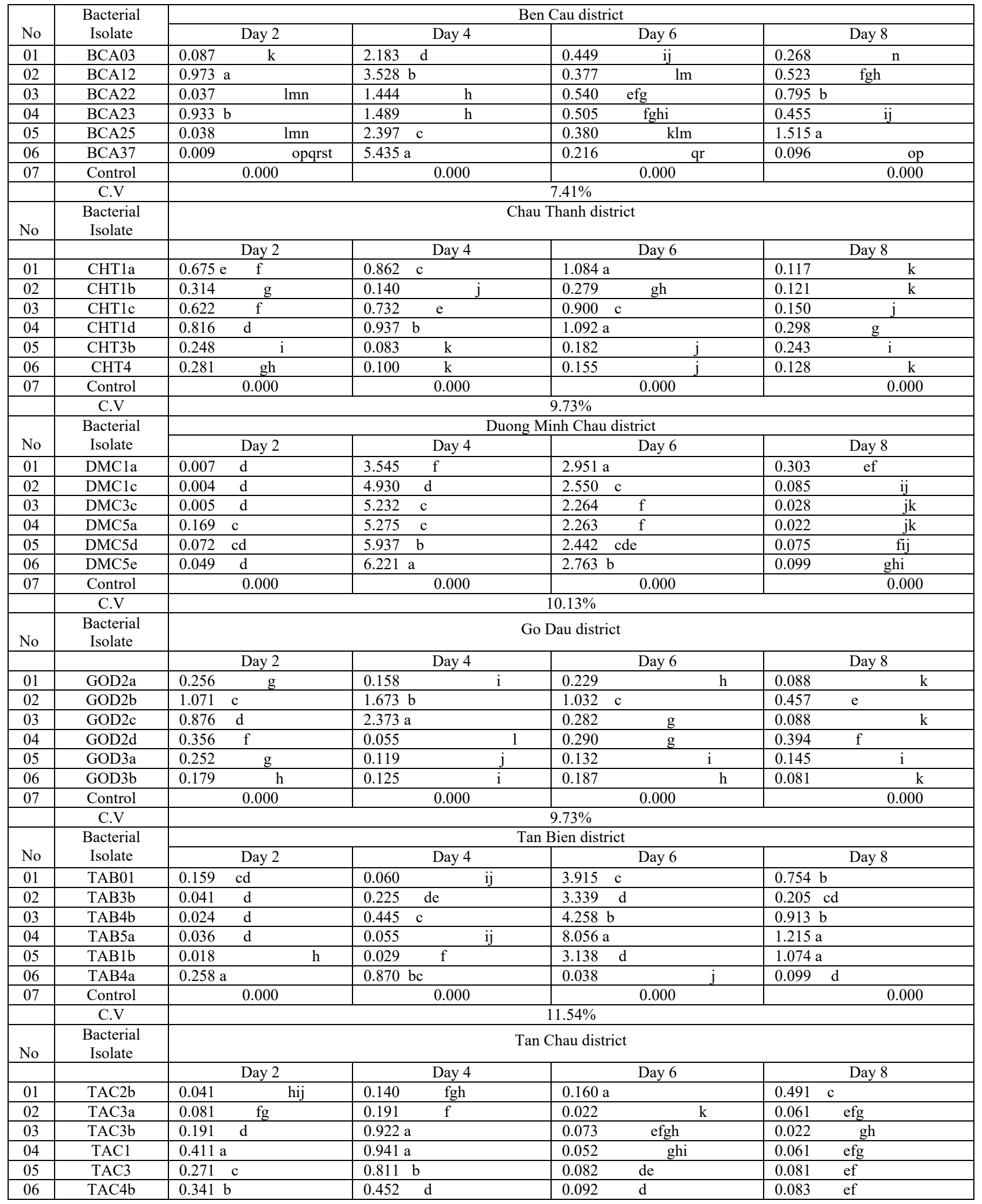




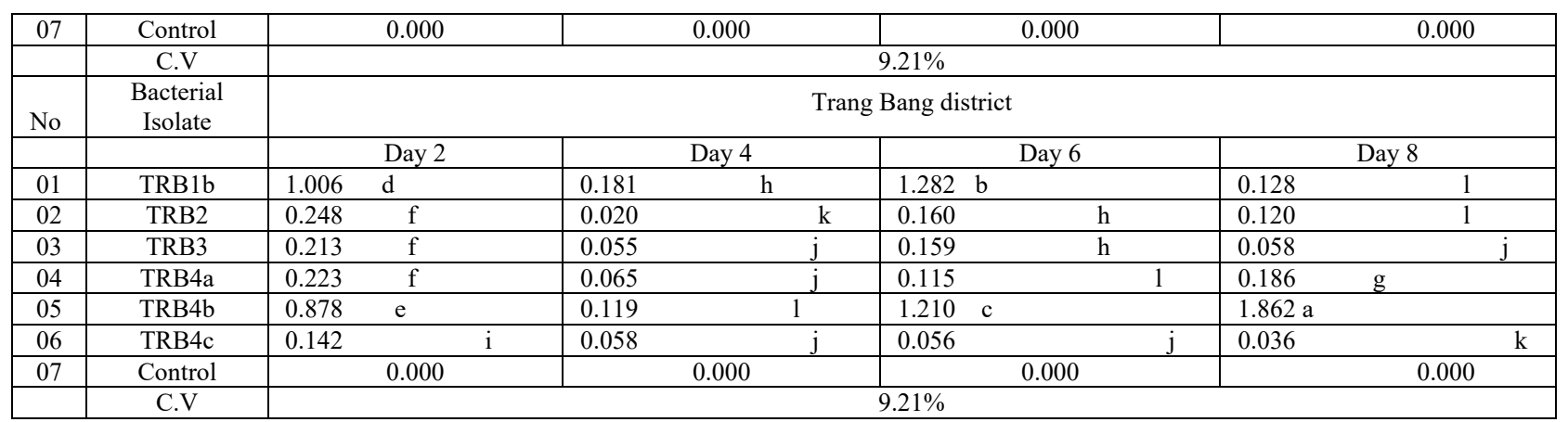

Means within a column followed by the same letter/s are not significantly different at $\mathrm{p}<0.01$

Table - 5 Phosphate solubilization $\left(\mathrm{mg} \mathrm{P}_{2} \mathrm{O}_{5} / \mathrm{ml}\right)$ of 70 isolates (10 isolates/site [or district]

\begin{tabular}{|c|c|c|c|c|c|}
\hline \multirow[t]{2}{*}{ No } & \multirow{2}{*}{$\begin{array}{l}\text { Bacterial } \\
\text { Isolate }\end{array}$} & \multicolumn{4}{|c|}{ Ben Cau district } \\
\hline & & Day 5 & Day 10 & Day 15 & Day 20 \\
\hline 01 & BCA06 & 93.35 & 104.92 & 238.65 & $327.00 \mathrm{~b}$ \\
\hline 02 & BCA07 & 81.38 & 082.13 & 146.10 & 150.16 \\
\hline 03 & BCA13 & 70.82 & 088.56 & 218.09 & 196.95 \\
\hline 04 & BCA17 & 82.66 & 079.09 & 149.11 & 147.34 \\
\hline 05 & BCA29 & 109.45 & 154.60 & 224.56 & 263.62 \\
\hline 06 & BCA30 & 59.53 & 118.77 & 225.35 & 287.00 \\
\hline 07 & BCA34 & 27.48 & $417.21 \mathrm{a}$ & 255.16 & 094.91 \\
\hline 08 & BCA37 & $158.17 \mathrm{a}$ & $360.33 \mathrm{~b}$ & 203.57 & 156.38 \\
\hline 09 & BCA38 & 85.88 def & $258.70 \mathrm{c}$ & 163.33 & 127.53 \\
\hline 10 & BCA39 & 84.18 ef & $262.52 \mathrm{c}$ & $388.14 \mathrm{a}$ & $385.57 \mathrm{a}$ \\
\hline 11 & Control & 0.00 & 0.00 & 0.00 & 0.00 \\
\hline & C.V & & & & \\
\hline
\end{tabular}

\begin{tabular}{|c|c|c|c|c|c|c|c|c|c|c|}
\hline \multirow[t]{2}{*}{ No } & \multirow{2}{*}{$\begin{array}{l}\text { Bacterial } \\
\text { Isolate }\end{array}$} & \multicolumn{9}{|c|}{ Chau Thanh district } \\
\hline & & \multicolumn{2}{|c|}{ Day 5} & \multicolumn{2}{|c|}{ Day 10} & \multicolumn{2}{|c|}{ Day 15} & \multicolumn{3}{|c|}{ Day 20} \\
\hline 01 & CHT2f & 19.57 & $\mathrm{j}$ & 100.15 & $\mathrm{f}$ & 134.91 & $\mathrm{e}$ & 193.68 & & \\
\hline 02 & CHT3a & 41.57 & $\mathrm{i}$ & 133.96 & e & 95.15 & $\mathrm{~g}$ & 83.96 & gh & \\
\hline 03 & CHT3b & 257.90 & & 114.26 & $\mathrm{f}$ & 100.60 & $\mathrm{f}$ & 59.20 & & $\mathrm{i}$ \\
\hline 04 & CHT3c & 77.38 & $\mathrm{~h}$ & 147.54 & & 175.63 & $\mathrm{~d}$ & 85.03 & gh & \\
\hline 05 & CHT4a & 16.31 & $\mathrm{j}$ & 99.71 & fg & 175.97 & $\mathrm{~d}$ & 197.86 & & \\
\hline 06 & CHT4c & 23.57 & $\mathrm{j}$ & 31.04 & $\mathrm{j}$ & 74.16 & $\mathrm{~h}$ & 82.67 & gh & \\
\hline 07 & CHT4f & 69.76 & $\mathrm{~h}$ & 92.60 & $\mathrm{~g}$ & 188.56 & & 112.98 & $\mathrm{f}$ & \\
\hline 08 & CHT1 & 197.05 & & 39.80 & $\mathrm{i}$ & 47.79 & $\mathrm{i}$ & 50.63 & & $\mathrm{i}$ \\
\hline 09 & CHT4d & 70.05 & $\mathrm{~h}$ & 25.0 & $\mathrm{j}$ & 104.67 & $\mathrm{f}$ & 132.25 & $\mathrm{e}$ & \\
\hline 10 & CHT4e & 54.67 & $\mathrm{i}$ & 78.07 & $\mathrm{~h}$ & 147.21 & $\mathrm{e}$ & 212.85 & & \\
\hline \multirow[t]{2}{*}{11} & Control & \multicolumn{2}{|c|}{0.00} & \multicolumn{2}{|c|}{0.00} & \multicolumn{2}{|c|}{0.00} & \multicolumn{3}{|c|}{0.00} \\
\hline & C.V & & & & & & & & & \\
\hline
\end{tabular}

\begin{tabular}{|c|c|c|c|c|c|c|}
\hline \multirow{2}{*}{ No } & \multirow{2}{*}{$\begin{array}{l}\text { Bacterial } \\
\text { Isolate }\end{array}$} & \multicolumn{5}{|c|}{ Duong Minh Chau district } \\
\hline & & Day 5 & Day 10 & Day 15 & & \\
\hline 01 & DMC1a & 20.59 & 52.41 & $95.04 \mathrm{a}$ & $112.26 \mathrm{a}$ & \\
\hline 02 & DMC1b & 25.24 & 42.64 & 41.05 & 31.66 & $\mathrm{j}$ \\
\hline 03 & DMC2a & 11.02 & 49.71 & $72.09 \mathrm{~b}$ & 73.48 & \\
\hline 04 & DMC2b & 22.48 & 35.45 & $53.64 \mathrm{c}$ & 61.62 & $\mathrm{~h}$ \\
\hline 05 & DMC3b & $31.47 \mathrm{c}$ & 27.96 & $50.08 \mathrm{~d}$ & 19.04 & 1 \\
\hline 06 & DMC5b & $15.22 \mathrm{e}$ & $106.21 \mathrm{a}$ & 1.03 & 39.28 & $\mathrm{i}$ \\
\hline 07 & DMC5d & 14.66 & 37.37 & 46.33 & 9.69 & $\mathrm{n}$ \\
\hline 08 & DMC5e & $47.02 \mathrm{~b}$ & 29.72 & 35.10 & 14.03 & $\mathrm{~m}$ \\
\hline 09 & DMC2c & $21.47 \quad \mathrm{~d}$ & $82.92 \mathrm{~b}$ & 45.28 & 72.29 & $\mathrm{f}$ \\
\hline 10 & DMC3 & 6.06 & 49.83 & 37.98 & $108.72 \mathrm{~b}$ & \\
\hline \multirow[t]{2}{*}{11} & Control & 0.00 & 0.00 & 0.00 & 0.0 & \\
\hline & C.V & \multicolumn{5}{|c|}{$9.14 \%$} \\
\hline
\end{tabular}


International Journal of Innovations in Engineering and Technology (IJIET) http://dx.doi.org/10.21172/ijiet.82.047

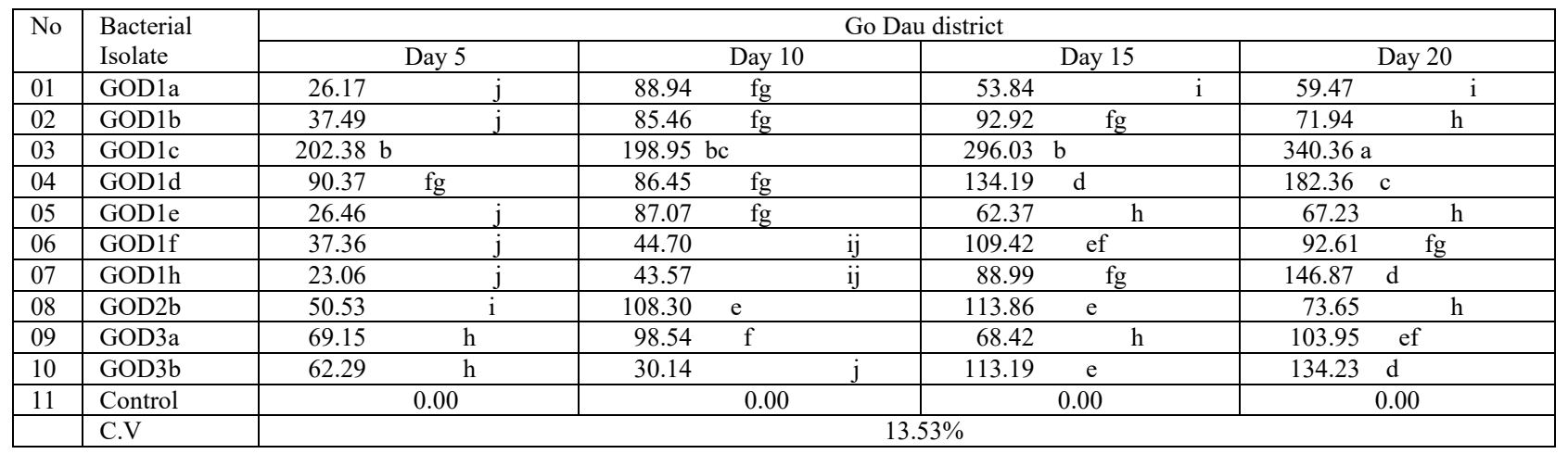

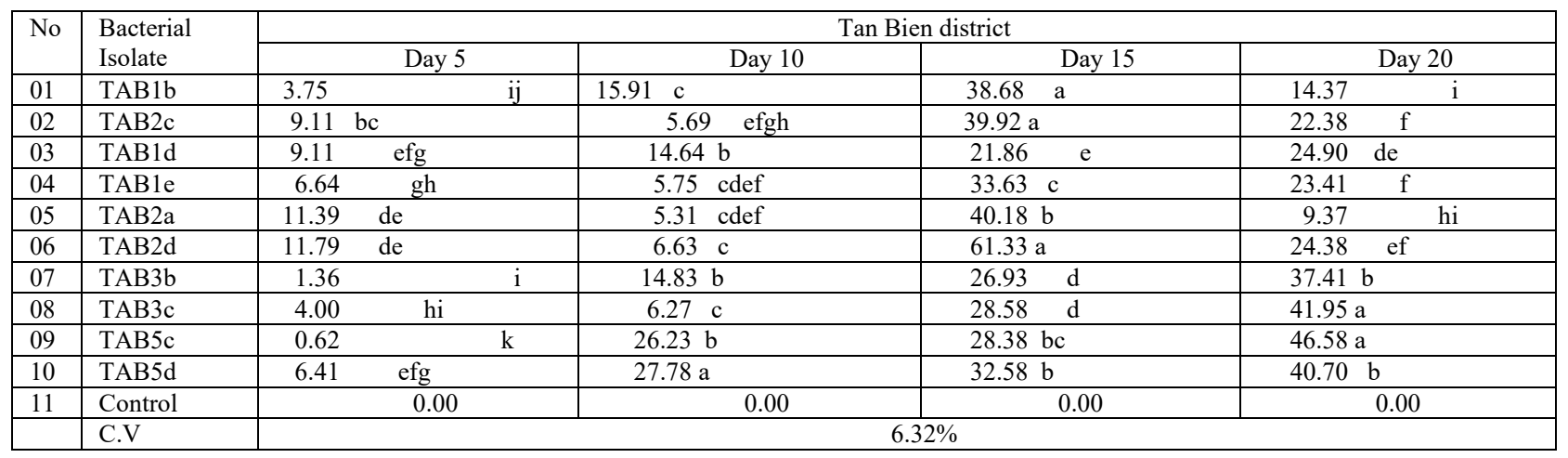

\begin{tabular}{|c|c|c|c|c|c|c|}
\hline \multirow[t]{2}{*}{ No } & \multirow{2}{*}{$\begin{array}{l}\text { Bacterial } \\
\text { Isolate }\end{array}$} & \multicolumn{5}{|c|}{ Tan Chau district } \\
\hline & & Day 5 & Day 10 & Day 15 & \multicolumn{2}{|c|}{ Day 20} \\
\hline 01 & TAC1c & 10.78 & 14.97 & $424.81 \mathrm{a}$ & 5.22 & $\mathrm{~h}$ \\
\hline 02 & TAC2c & 3.70 & 131.31 & 195.89 & 346.09 & \\
\hline 03 & TAC3a & $46.64 \mathrm{bc}$ & $613.12 \mathrm{a}$ & $404.19 \mathrm{ab}$ & 3.83 & $\mathrm{~h}$ \\
\hline 04 & TAC $3 \mathrm{~b}$ & $54.37 \mathrm{ab}$ & $651.54 \mathrm{a}$ & $428.40 \mathrm{a}$ & 3.56 & $\mathrm{~h}$ \\
\hline 05 & TAC3c & $55.67 \mathrm{ab}$ & $533.33 \mathrm{~b}$ & $353.35 \mathrm{c}$ & 5.47 & $\mathrm{~h}$ \\
\hline 06 & TAC4a & $54.92 \mathrm{ab}$ & $529.94 \mathrm{~b}$ & $414.95 \mathrm{ab}$ & 378.94 & \\
\hline 07 & TAC4b & $51.89 \mathrm{ab}$ & $547.01 \mathrm{~b}$ & $315.40 \quad \mathrm{~d}$ & 4.60 & $\mathrm{~h}$ \\
\hline 08 & TAC4c & $61.25 \mathrm{a}$ & $406.94 \mathrm{c}$ & 277.43 & 3.34 & $\mathrm{~h}$ \\
\hline 09 & TAC4d & 6.27 & $254.25 \mathrm{~d}$ & 383.11 bc & 37.94 & \\
\hline 10 & TAC4a & 34.74 & 28.94 & $195.26 \mathrm{f}$ & $492.74 a$ & \\
\hline 11 & Control & 0.00 & 0.00 & 0.00 & & \\
\hline & C.V & & & & & \\
\hline
\end{tabular}

Means within a column followed by the same letter/s are not significantly different at $\mathrm{p}<0.05$

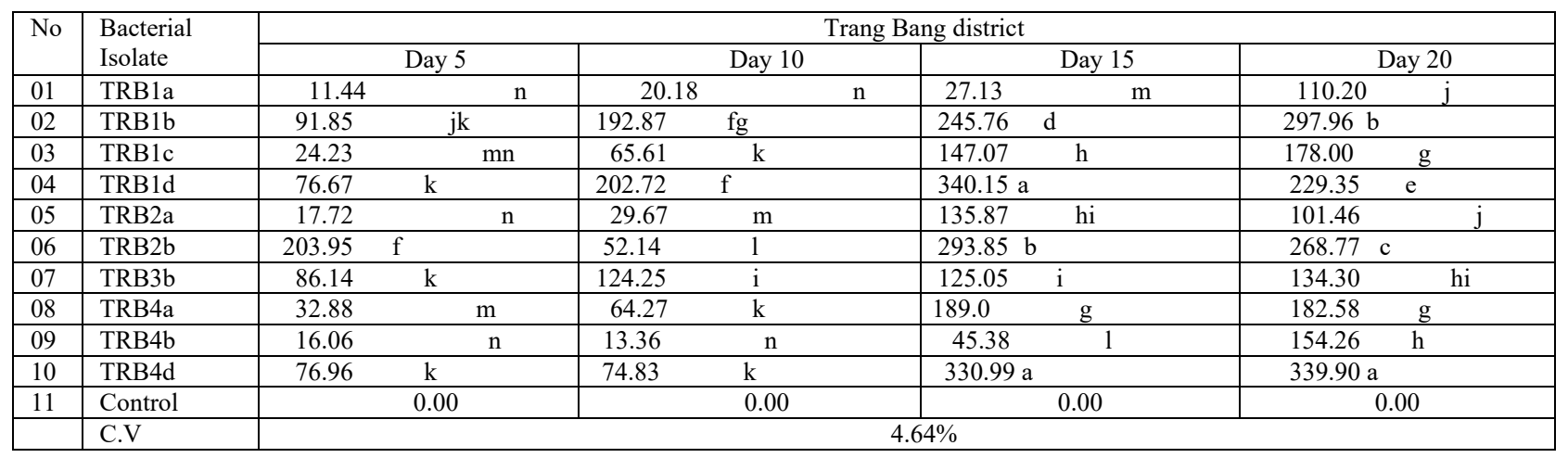




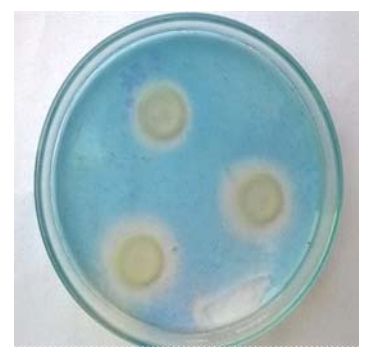

A

A: BCA07 isolate

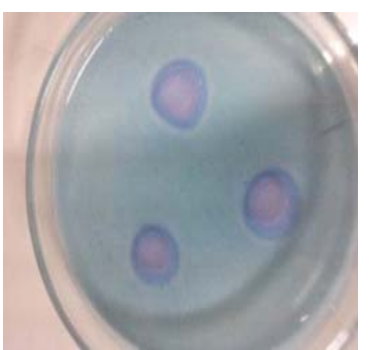

B

B: DMC5e isolate

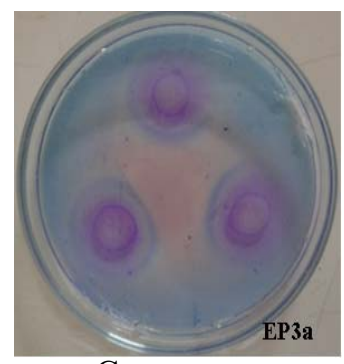

$\mathrm{C}$

$\mathrm{C}$ : TAB3b isolate

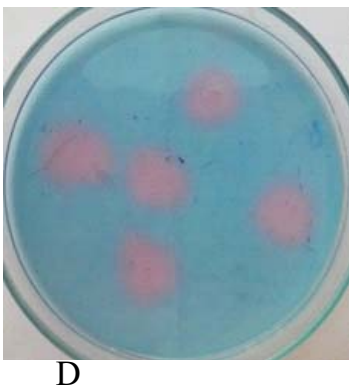

D: CHT2b isolate

Figure - 5 Bacterial isolates made a yellow, orange halo round well contaning bacterial liquid on CAS agar after $48 \mathrm{~h}$ incubation

Based on the characteristics as high nitrogen fixation, phosphate solublization, IAA and siderophores, 23 good isolates were chosen to identify with universal primers $27 \mathrm{~F}$ and $1492 \mathrm{R}$ and sequencing as BCA07, BCA17, CHT1a, CHT1d, CHT2f, CHT4c, CHT4e, DMC1a, DMC2a, DMC2c, DMC5e, GOD1c, GOD1f, GOD2c, GOD2f, TAB01, TAB5d, TAC3b, TAC4a, TRB1b, TRB1d, TRB2b, TRB4d.

The fragment of $1485 \mathrm{bp} 16 \mathrm{~S}$ rRNA were obtained from PCR with 27F and 1492R primers and sequencing. Homology searches of 16S rRNA gene sequence of selected strain in GenBank by BLAST revealved that they had similarity to sequences of Bacilli (6/23 isolates), 4 isolates belonged to Betaproteobacteria, 13 strains were Gammaproteobacteria (Figure 4) (Table 6).

Table - 6 Phylogenetic affiliation of isolates on the basis of 16S rRNA genes sequences by using BLAST programme in the GenBank database based on sequences similarity

\begin{tabular}{|c|c|c|}
\hline Taxonomic group and strain & Closest species relative & Similarity $(\%)$ \\
\hline \multicolumn{3}{|l|}{ Bacilli } \\
\hline \multirow[t]{2}{*}{$\mathrm{DNC} 2 \mathrm{c}$} & Bacillus subtilis strain L23 (KU179336) & 99 \\
\hline & Bacillus methylotrophicus strain JF29 (KC172004) & 99 \\
\hline \multirow[t]{2}{*}{ TAB5d } & Bacillus aryabhattai strain TC1-29 (KY673685) & 98 \\
\hline & Bacillus flexus strain JIA2 (KX607116) & 98 \\
\hline \multirow[t]{2}{*}{ BCA17 } & Bacillus subtilis strain W1-3 (KY368671) & 100 \\
\hline & Streptomyces sp. strain AP42 (KY608579) & 100 \\
\hline \multirow[t]{2}{*}{ GOD1c } & Bacillus subtilis strain B18 (KJ870198) & 99 \\
\hline & Bacillus tequilensis strain EGY-WCP11 (KF562338) & 99 \\
\hline \multirow[t]{2}{*}{ CHT1a } & Bacillus subtilis strain PR38 (KJ870046) & 99 \\
\hline & Bacillus tequilensis strain HQB660 (KT758573) & 99 \\
\hline \multirow[t]{2}{*}{ CHT1d } & Bacillus subtilis strain N-11 (GQ452910) & 99 \\
\hline & Bacillus amyloliquefaciens strain CC1HG7 (KU564242) & 98 \\
\hline \multicolumn{3}{|l|}{ Betaproteobacteria } \\
\hline \multirow[t]{2}{*}{ DMC5e } & Burkholderia sp. STJ14 (KC833509) & 99 \\
\hline & Burkholderia seminalis strain IHB B 15122 (KM817204) & 99 \\
\hline \multirow[t]{2}{*}{ TAC3b } & Burkholderia sp. B2(2014) (KM054695) & 98 \\
\hline & Burkholderia latens strain R-5630 (KX345793) & 98 \\
\hline \multirow[t]{2}{*}{$\mathrm{DMC} 2 \mathrm{a}$} & Burkholderia vietnamiensis strain B3 (AY741147) & 97 \\
\hline & Burkholderia latens strain R-5630 (NR_042632) & 97 \\
\hline \multirow[t]{2}{*}{ BCA07 } & Burkholderia sp. strain S6-1 (KY357342) & 99 \\
\hline & Burkholderia vietnamiensis strain MSMB608WGS (CP013456) & 99 \\
\hline
\end{tabular}




\begin{tabular}{|c|c|c|}
\hline TAB01 & Acinetobacter calcoaceticus strain NCTC7364 (LT605059) & 99 \\
\hline & Acinetobacter sp. strain M05 (KT964806) & 99 \\
\hline \multirow[t]{2}{*}{ TAC4a } & Acinetobacter sp. 150 (KC257011) & 99 \\
\hline & Acinetobacter baumannii strain HBf01 (KJ646022) & 99 \\
\hline \multirow[t]{2}{*}{ DMC1a } & Acinetobacter sp. TW (FJ753401) & 99 \\
\hline & Acinetobacter baumannii strain CGK-W8 (HM485467) & 99 \\
\hline \multirow[t]{2}{*}{ TRB1b } & Acinetobacter sp. IHB B 6803 (KF668456) & 99 \\
\hline & Acinetobacter calcoaceticus strain ATCC 23055 (NR_119357) & 99 \\
\hline \multirow[t]{2}{*}{ CHT4e } & Acinetobacter sp. JH250-8 (HQ638093) & 99 \\
\hline & Acinetobacter seifertii strain LUH 1472 (NR_134684) & 99 \\
\hline \multirow[t]{2}{*}{$\mathrm{CHT} 4 \mathrm{c}$} & Acinetobacter sp. YL3 (KR912218) & 99 \\
\hline & Acinetobacter calcoaceticus strain B40 (JX010982) & 99 \\
\hline \multirow[t]{2}{*}{ TRB1d } & Acinetobacter calcoaceticus strain ATCC 23055 (NR_119357) & 99 \\
\hline & Acinetobacter bereziniae strain MBT3 (JX966440) & 99 \\
\hline \multirow[t]{2}{*}{ GOD2c } & Acinetobacter calcoaceticus strain EU04 (JF681282) & 98 \\
\hline & Acinetobacter seifertii strain LUH 1472 (NR_134684) & 97 \\
\hline \multirow[t]{2}{*}{ TRB2b } & Acinetobacter calcoaceticus strain M.pstv.12.3 (KM108497) & 99 \\
\hline & Acinetobacter seifertii, strain: 2pv (LC191524) & 99 \\
\hline \multirow[t]{2}{*}{ TRB4d } & Acinetobacter sp. 156 (KC257016) & 100 \\
\hline & Acinetobacter nosocomialis strain PJ1M2 (KU320983) & 100 \\
\hline \multirow[t]{2}{*}{ GOD2f } & Enterobacter cloacae strain UKME02 (KX266260) & 99 \\
\hline & Enterobacter hormaechei strain RPK2 (KX980424) & 99 \\
\hline \multirow[t]{2}{*}{ CHT2f } & Enterobacter cloacae strain UKME02 (KX266260) & 99 \\
\hline & Enterobacter xiangfangensis strain LMG27195 (CP017183) & 99 \\
\hline \multirow[t]{2}{*}{ GOD1f } & Enterobacter cloacae strain PCX2 (KU936831) & 99 \\
\hline & Enterobacter hormaechei strain RCT10 (HM771693) & 99 \\
\hline
\end{tabular}

A maximum-likelihood analysis of phylogenetic tree in these isolates showed in the two clusters: Cluster A had two smaller clusters including cluster A1 and A2. According to cluster A1, A11 had two strains such as Bacillus subtilis BCA17 and Acinetobacter calcoaceticus TRB2b in cluster A111 and Cluster A112 with two strains Acinetobacter calcoaceticus GOD2c showed close relationship with Acinetobacter sp. TRB4d, while cluster A12 having three strains as Burkholderia vietnamiensis DMC2a, especially two strains Acinetobacter sp. DMC1a and Acinetobacter sp. TRB1b related very closely. Furthermore, all of them (strains in cluster A11, A12 and A21) related with strain Bacillus subtilis GOD1c (Figure 7). In cluster A2, cluster A21 composed of strain Burkholderia sp. BCA07, Acinetobacter sp. CHT4e and Burkholderia sp. TAC3b were isolated soils of three other districts but they had close relationship while cluster A22 with two strains Enterobacter cloacae GOD2f and Enterobacter cloacae CHT2f had relationship closely with strain Bacillus subtilis CHT1a. All of them had relationship with strain Bacillus aryabhattai TAB5d. 


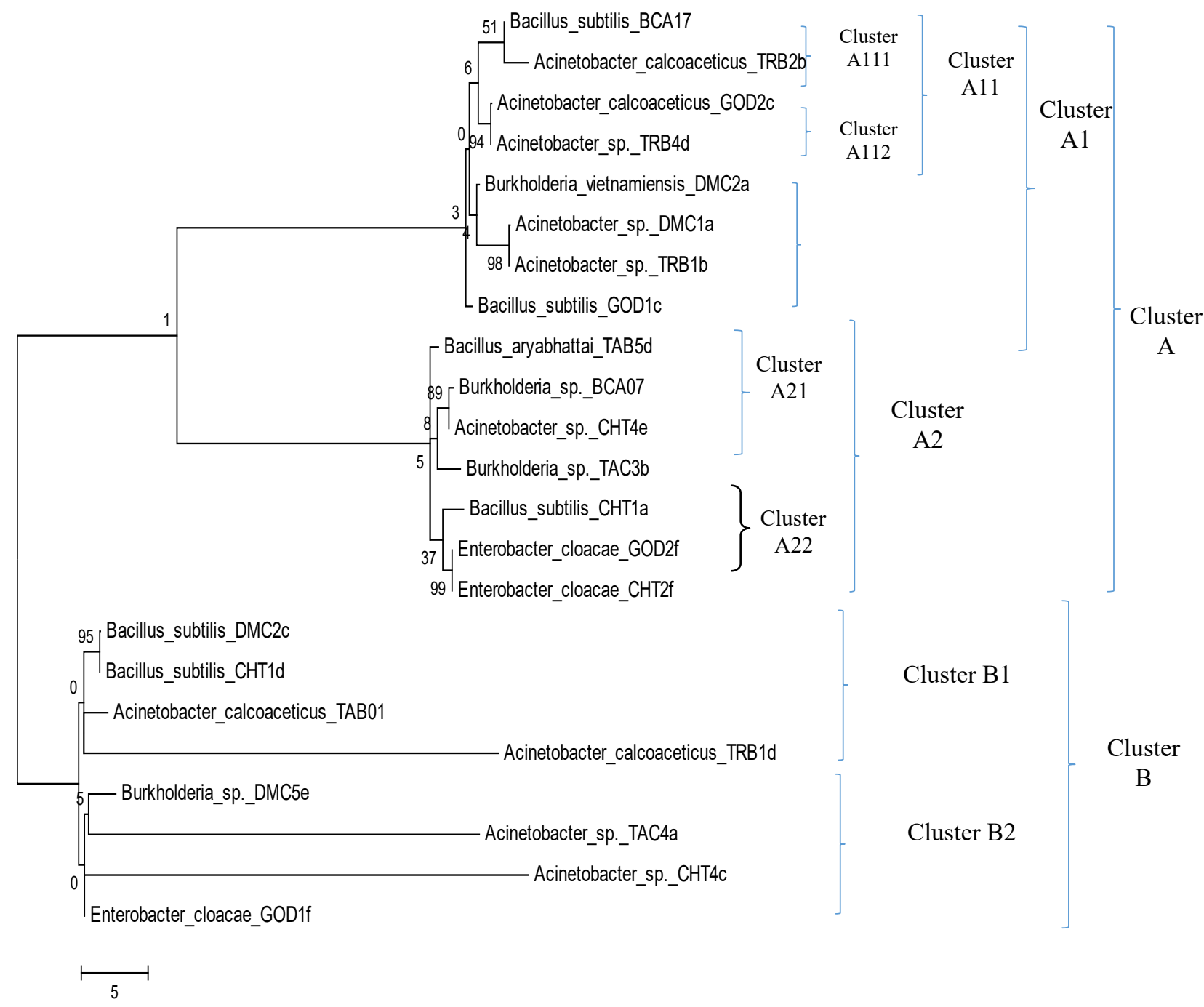

Figure - 4 Phylogenetic tree showing the relative position of rhizobacteria (PGPR) by the maximum-likelihood method of complete 16S rRNA sequences. Bootstrap value values of 1000 replicates are shown at the nodes of the trees.

In cluster B composed of two small clusters as cluster B1 with two strains Bacillus subtillus DMC2c and Bacillus subtillus CHT1d with high homology (95\%) and two strains Acinetobacter calcoaceticus TAB01 and Acinetobacter calcoaceticus TRB1d. This result showed that the strains originating from Tan Bien district, Duong Minh Chau district had close relationship with strains isolating from soil of Chau Thanh district and Trang Bang district even though four these districts are far over100 km. In small cluster B2 included four strains Burkholderia sp. DMC5e, Acinetobacter sp. TAC4a, Acinetobacter sp. CHT4c and Enterbacter cloacae GOD1f. Whereas all of them belong to proteobacteria, Burkholderia sp. DMC5e was Beta-proteobacteria, and three strains were Gammaproteobacteria with two strains Acinetobacter sp. TAC4a and Acinetobacter sp. CHT4c showing close relationship even though they were isolated from soil of Tan Chau district and Chau Thanh district.

Among 23 strains, there were 16 strains had length nucleotide (over 600) and Theta values (per sequence) from $\mathrm{S}$ of SNP for DNA polymorphism were calculated for Each group, and Gammaproteobacteria group had the highest values as comparison with Betaproteobacteria and Bacilli (Table 7). 
Table - 7 Genetic diversity of 16 strains

\begin{tabular}{lccc}
\hline & Nucleotie diversity & Theta (per site) from Eta & Theta (per site) from S $(\boldsymbol{\theta})$ \\
\hline 16 strains & 0.73124 & $0.86103 \pm 0.103$ & $0.29579 \pm 0.0107$ \\
\hline Primers 27F and 1492R & &
\end{tabular}

Primers 27F and 1492R

The rhizospheric bacteria have been studied and described as beneficial bacteria with Gammaproteobacter presented on both Burk's and NBRIP medium and it occupied over 50\% in the total of 23 strains according to our result (Figure 5).

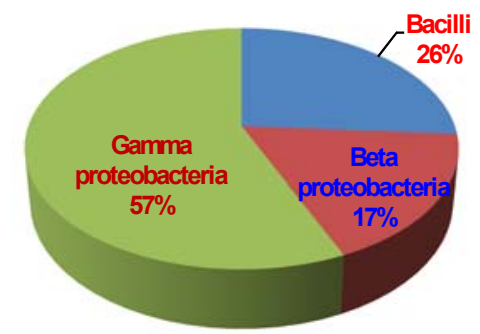

Figure - 5 The proportion of group and they distributed in two clusters

The plant-beneficial rhizobacteria may decrease the global dependence on hazardous agricultural chemicals which destabilize the agro-eco-systems [21]. The plant growth promoting rhizobacteria (PGPR), are characterized by the following inherent distinctiveness: (i) they must be proficient to colonize the root surface (ii) they must survive, multiply and compete with other microbiota, at least for the time needed to express their plant growth promotion/protection activities, and (iii) they must promote plant growth [22]. 'Bacilli' AEFB are a diverse group with wide distribution in agricultural soils that contribute both directly and indirectly to plant development [23]. New pecies of aerobic endospore forming bacteria (AEFB) have been isolated from sugarcane internal tissues and rhizosphere [24][25][26]. The our result of previous experiment carried out on acrisols of Dong Nai province (nearly Tay Ninh province), occureance of genus Bacillus in soil cultivated sugarcane with high nitrogen fixation and phosphate solubilization ability [27] and Chinese scientists isolated many species of Enterobacter and Klebsiella having high nitrogen fixation, phosphate solubilization, IAA biosynthesis and siderophores production in soil cultivating sugarcane on Guangxi province, China [28](Lin et al., 2012). We isolated 331 bacterial isolates from 41 acrisols samples of Tay Ninh province and selected of 23 good strains from seven sites (districts) to identify and sequence. From 23 good strains, four strains (Burkholderia sp. DMC5e, Bacillus subtilis GOD1c, Bacillus subtilis CHT1d and Burkholderia sp. TAC3b) revealed promising candidates with multiple beneficial characteristics and they have the potential for application as inoculants adapted to poor soils and sugar cultivation in province.

\section{IV.CONCLUSION}

From 36 soil samples of acrisols of sugarcane regions in seven districts (sites) of Tay Ninh province, the SouthEastern Vietnam, 331 isolates were isolated on two media as Burk's N free and NBRIP. Besides, they were identified as rhizospheric bacteria and 23 isolates having good plant growth promotion were chosen to analyse their relationship. These isolates were identified as Gammaproteobacteria (more than 50\%), Bacilli (26\%) and Betaproteobacteria (17\%) in acrisols. Among them, four strains should be recommended to test their effectiveness in sugarcane in-vitro.

\section{REFERENCES}

[1] J.L. Baldani, V.M. Reis, V.L.D. Baldani and J. Dobereiner, "A brief story of nitrogen fixation in sugarcane - reasons for success in Brasil,” Funct. Plant. Biol., vol.29, pp.417-423, 2002.

[2] L.H.A. Thi, "Report of Sugarcane production in 2012 (Vietnamese)," Department of Agriculture and Rural Development, Vietnam 2013.

[3] N. Tejera, C. Lluch, M.V. Martinez-Toledo and J. Gonzalez-Lopez,'Isolation and characterization of Azotobacter and Azospirillum strains from the sugarcane rhizosphere," Plant Soil., vol. 270, pp:223-232, 2005. 
[4] N.D.Quang, C.A.Duong, T.T.M.Dung and D.C. Thong, "Process of Sugarcane Cultivation in the eastern of South Vietnam (Vietnamese)," Department of Agriculture and Rural Development, Vietnam, 2010.

[5] V.A. Duong "Báo cáo chuyên đề: Thực Trạng sản xuất, yếu tố hạn chế và đề xuất gói kỹ thuật sản xuất mía năng suất chất lượng cao cho vùng Đông Nam Bộ. Hội thảo định hướng nghiên cứu phát triển mía đường bền vững ở Việt Nam, ngày 29/10/2014" (Vietnamese).

[6] T.S. Walker, H.P. Bais, E. Grotewold, and V.M. Vivanco, "Root exudation and rhizosphere biology," Plant Physiol., vol.132, pp. 44-51, 2003.

[7] J.W. Kloepper, W. Mahafee, J.A. McInroy and P.A. Beckman, "Comparative analysis of methods for recovering plant growth-promoting rhizobacteria from roots," Can. J. Microbiol., vol. 39, pp.953-957, 1991.

[8] M. Ahemad and M. Kilbert, "Mechanisms and applications of plant growth Promoting rhizobacteria: Current perspective,". Journal of King Saud University - Science. http://dx.doi.org/10.1016/j.jksus. 2013.05.001.

[9] H.J. Hoben, and P. Somasegaran, "Comparison of Pour, Spread and Drop Plate Methods for Enumeration of Rhizobium spp. In Inoculants made from presterilized peat,” Appl. Environ. Microbiol., vol. 44, pp. 1246-1247, 1982.

[10] M. Park, C. Kim, J. Yang, H. Lee, W. Shin, S. Kim and T. Sa, "Isolation and characterization of diazotrophic growth promoting bacteria from Gram rhizosphere of agricultural crops of Korea," Microbiological Research, vol 160, pp. 127-133, 2005.

[11] C.S. Nautiyal, "An efficient microbiological growth medium for screening phosphate-solubilizing microorganisms," FEMS Microbiology Letters, vol. 170, pp.256-270, 1999.

[12] S.A. Gordon and R.P. Weber., "Colometric estimation of indolacetic acid," Plant Physiol., vol 26, pp.192-195, 1951.

[13] B., Schwyn, and J.B. Neilands. "Universal chemical assay for the detection and determination of siderophores". Analytical Biochemistry, vol. 60(1), pp. 47-56, 1987.

[14] J. Murphy and J. P. Riley, "A modified single solution for determination of phosphate in natural waters," Anal. Chim. Acta., vol. 27, pp. 31$36,1962$.

[15] J.M. Andersen and J.S.I. Ingram, “Tropical Soil Biology and Fertility: A Handbook on Methods, 2 nd edn," CAB International Wallingford, Oxford, UK, 221, p. 1993.

[16] B. Neumann, A. Pospiech, and H.U. Schairrer, "Rapid isolation of genomic DNA from Gram-negative," Trends Gent., vol. 8, pp. 332$333,1992$.

[17] S. Turner, K.M. Pryer, V.P.M. Miao, and J.D. Palmer, "Investigating deep phylogenetic relationships among cyanobacteria and plastids by small subnit rRNA sequence analysis," J. Eukaryotic Microbiol., vol. 46, pp. 327-338, 1999.

[18] K. Tamura, D. Peterson, N. Peterson, G Stecher, M. Nei, and S. Kumar, "MEGA5: Molecular Evolutionary Genetics Analysis using Maximum Likehood, Evolutionary Distance and Maximum Parsimony Methods," Mol. Biol. Evol., vol. 28, pp. 2731-2739, 2011.

[19] M.K. Halushka, J.B. Fan, K. Bentley, L. Hsie, N. Shen, A. Weder, R. Cooper, R. Lipshutz, and A. Charavarti, "Patterns of single-nucleotide polymorphisms in candidate genes for blood-pressure homestasis," Nat. Genet., vol.22(3), pp.239-247, 1999.

[20] D.T..N. Thanh and C.N. Diep, "Isolation and Identification of rhizospheric bacteria in Acrisols of maize (Zea mays L.) in the eastern of South Vietnam. American J. Life Science. Vol. 2, No. 2, 2014, pp. 82-89. doi: 10.11648/j.ajls.20140202.18.

[21] M. Ahemad and M. Kilbert, "Mechanisms and applications of plant growth Promoting rhizobacteria: Current perspective,". Journal of King Saud University - Science. http://dx.doi.org/10.1016/j.jksus. 2013.05.001.

[22] J.W. Kloepper, "Plant growth-promoting rhizobacteria (other systems). In: Y. Okon. (Ed.) Azospirillum/Plant Associations," CRC Press, Boca Raton, FL. USA, pp. 111-118, 1994.

[23] B.B. McSadden, "Ecology of Bacillus and Paenabacillus spp. in Agricultural Systems," Phytopathol., vol.94, pp. 1252-1258, 2004.

[24] L.A. Mirabal, E. Ortega, R. Rodes, L. Fernandez, and E. Perez, "Another nitrogen-fixing microorganisms in sugarcane stalks: bacillus brevis," Cultiv. Tropicales, vol.21(4), pp. 9-12, 2000.

[25] R. Rivas, P. Garcia-Fraile, J.L. Zurdo-Pineiro, P.F. Mateos, E. Martinez-Molina, E.J. Bedmar, J. Sanchez-Raya, and E. Velaquez, "Saccharibacillus sacchari gen. nov. sp. nov., isolated from sugarcane,: Int. J. Syst. Evol. Microbiol., vol. 58, pp. 1850-1854, 2008.

[26] M. Madhaiyan, S. Poonguzhali, J.S. Lee, K.C. Lee, and K. Hari, "Bacillus rhizosphaerae sp. nov., a novel diazotrotrophic bacterium isolated from sugarcane rhizosphere soil," Antonie Van Leeuwenhoek, vol. 100, pp. 437-444, 2011.

[27) H.M. Tam and C.N. Diep, "Isolation, Characterization and Identification of Endophytic Bacteria in Sugarcane (Saccharum spp. L.) Cultivated on Soils of the Dong Nai province, Southeast of Vietnam," American J. Life Science, vol.2, No.2 2014, pp. 361-368. doi: 10.11648/j.ails.2014206.16.

[28) L. Lin, Z. Li, C. Hu, X. Zhang, S. Chang, L. Yang, Y. Li, and Q. An. Plant growth-promoting nitrogen-fixing enterobacteria are in association with sugarcane plants growing in Guangxi, China. Microbes Environ, vol. 27(4),pp. 391-8, 2012. 